\title{
Association between Thyroid-Stimulating Hormone Levels and Non-Alcoholic Fatty Liver Disease Is Not Independent from Metabolic Syndrome Criteria
}

\author{
Carolina Castro Porto Silva Janovsky ${ }^{a, b}$ Fernando H. Cesena ${ }^{a}$ \\ Viviane Arevalo Tabone Valente ${ }^{a}$ Raquel Dilguerian de Oliveira Conceição ${ }^{a}$ \\ Raul D. Santos ${ }^{a, c}$ Márcio Sommer Bittencourt ${ }^{a, d, e}$ \\ ${ }^{a}$ Hospital Israelita Albert Einstein, Sao Paulo, Brazil; b Department of Medicine, Thyroid Outpatient Clinic, Division of \\ Endocrinology, Escola Paulista de Medicina, Universidade Federal de São Paulo, Sao Paulo, Brazil; ' Lipid Clinic Heart \\ Institute (InCor), University of Sao Paulo Medical School Hospital, Sao Paulo, Brazil; ${ }^{d}$ School of Medicine, Faculdade \\ Israelita de Ciências da Saúde Albert Einstein, Sao Paulo, Brazil; ' ${ }^{\circ}$ Center for Clinical and Epidemiological Research, \\ University Hospital, University of São Paulo School of Medicine, Sao Paulo, Brazil
}

\section{Keywords}

Thyroid function - Obesity · Non-alcoholic fatty liver disease . Metabolic syndrome . Thyroid-stimulating hormone

\begin{abstract}
Introduction: Thyroid hormones are involved in the regulation of body composition, lipid metabolism, and insulin resistance. Thus, it is possible that they might play a role in the pathogenesis of non-alcoholic fatty liver disease (NAFLD). However, the role of thyroid function on NAFLD is not well defined. In this study, we evaluated the relationship between thyroid-stimulating hormone (TSH) levels, within the reference range, and presence of NAFLD in asymptomatic individuals. Study Design: We included all individuals evaluated at a preventive clinic of the Hospital Israelita Albert Einstein, between 2014 and 2015. The prevalence of NAFLD (analyzed by abdominal ultrasound), according to TSH quartiles, within the reference range, was determined. The association between TSH quartiles and NAFLD was analyzed by logistic regression adjusted for possible confounders. Results: We evaluated 10,539 individuals (73\% male, age
\end{abstract}

$43.4 \pm 9.4$ years). The prevalence of NAFLD was $34,38,38$, and $39 \%$ in the first to the fourth TSH quartiles $(0.46-1.44$, $1.45-1.97,1.98-2.68$, and $2.69-4.68 \mathrm{mUI} / \mathrm{L}$, respectively, $p$ for trend $<0.001)$. At univariate analysis, higher TSH levels were associated with the diagnosis of NAFLD. When data were adjusted for the metabolic syndrome characteristics (waist circumference, HDL-cholesterol and triglycerides levels, presence of diabetes, and systemic arterial hypertension), the association was no longer significant. Conclusions: Although the TSH variability within the reference range is associated with NAFLD in univariable models, once adjusted for metabolic syndrome factors no significant association is noted.

(c) 2018 European Thyroid Association Published by S. Karger AG, Basel

\section{Introduction}

Thyroid hormones are involved in the regulation of body composition, lipid metabolism, and insulin resistance [1-6]. It is well known that hypothyroidism is associated with high levels of LDL-cholesterol and triglyc-

\section{KARGER}

E-Mail karger@karger.com

www.karger.com/etj (c) 2018 European Thyroid Association.

Published by S. Karger AG, Basel
Carolina Castro Porto Silva Janovsky

Hospital Israelita Albert Einstein

Avenida Brasil, 953 - Jardim Paulista

Sao Paulo, SP 01431-000 (Brazil)

E-Mail carolina.janovsky@ gmail.com 
erides secondary to a decrease in LDL receptor's activity and clearance of triglyceride-rich lipoproteins [7-9].

Excessive triglyceride accumulation in the liver is manifested through the diagnosis of steatosis, a component of non-alcoholic fatty liver disease (NAFLD) or non-alcoholic steatohepatitis $[10,11]$. In fact, NAFLD has long been recognized as the hepatic feature of the metabolic syndrome [12]. This condition may lead to hepatic fibrosis, cirrhosis, and hepatocellular carcinoma if not treated in the long term [13-16], and might be considered an independent risk marker for cardiovascular disease [17-19].

Previous studies have sought to evaluate the association between hypothyroidism and NAFLD [20-25]. Most of them showed that hypothyroidism, in its overt or subclinical type, is independently associated to NAFLD [26, 27], suggesting that hypothyroidism may directly result in NAFLD irrespective of other common metabolic risk factors. In a recent prospective study, lower thyroxine (T4) levels were associated with a higher risk for NAFLD [26]. Nevertheless, though this association has been demonstrated for subclinical hypothyroidism, the thyroid function is a continuum and the association between thyroid-stimulating hormone (TSH) levels within the normal range and NAFLD has not been well established. In the present study, we aimed to evaluate the association between thyroid function within the normal range and the presence of NAFLD in asymptomatic individuals.

\section{Methods}

All individuals evaluated at a routine health check-up at the Hospital Israelita Albert Einstein, between 2014 and 2015, were included. This evaluation comprised several clinical and laboratory tests, as well as abdominal ultrasound. The Ethical Committee of the Hospital Israelita Albert Einstein approved this study, and the consent form was waived as the research is based on database analysis and no additional patient contact was needed.

Individuals between 20 and 80 years, whose TSH levels were within the normal range, were evaluated, excluding those with previously known liver or thyroid disease, using thyroid-related medication (levothyroxine, methimazole or propylthiouracil), and with a score of more than 8 points in the Alcohol Use Disorders Test [28].

The check-up protocol comprised anthropometric evaluation, laboratory measures, physical activity questionnaire, and abdominal ultrasound for NAFLD evaluation, as previously described [29]. Waist circumference was measured by a trained nutritionist and was considered abnormal when above $94 \mathrm{~cm}$ for men and $80 \mathrm{~cm}$ for women. Body mass index (BMI) was measured using the weight/ height ${ }^{2}\left(\mathrm{~kg} / \mathrm{m}^{2}\right)$ formula. Plasma concentrations of total cholesterol, high-density lipoprotein-cholesterol (HDL-C), and triglycerides were determined by using automated equipment VITROS $5600^{\circledR}$ Ortho Clinical Diagnostics by dry chemical colorimetric method. Serum TSH was measured by a chemo luminescence method. Individuals were then divided into 4 groups according to quartiles of TSH levels within the reference range (0.46-1.44, 1.45$1.97,1.98-2.68$, and $2.69-4.68 \mathrm{mUI} / \mathrm{L}$ ). The international physical activity questionnaire (iPAQ) was used to evaluate the physical activity level in the last week [30]. The presence of NAFLD defined as hepatic steatosis in the absence of excessive alcohol consumption or previous liver disease was based on the ultrasound evaluation, according to standardized criteria [31]. Steatosis was diagnosed by certified radiologists after at least a 6-h fast using an ACUSON XP-10 device (Mountain View, CA, USA) and was identified by the presence of an ultrasonographic pattern of a bright liver, with evident contrast between hepatic and renal parenchyma.

\section{Statistical Analysis}

Continuous variables were presented as means and standard deviations or medians and quartiles, as appropriate, and compared using one-way ANOVA or Kruskal-Wallis test. Categorical variables were presented as absolute numbers and proportions, and compared using chi-square test. Triglycerides and TSH were logtransformed for inclusion in the multivariable models as these variables are known to have a skewed distribution.

The association between TSH levels and presence of NAFLD was analyzed using logistic regression models. Initially, we performed a univariable model followed by additional multivariable models adjusted as follows:

- Model 1: adjusted for gender and age;

- Model 2: adjusted for gender, age, smoking, and metabolic syndrome criteria (abdominal circumference, triglycerides, HDL-C, blood pressure, fasting glucose as continuous variables);

- Model 3: adjusted for gender, age, smoking, metabolic syndrome criteria (as in model 2), and physical activity levels.

We first performed those models using TSH as a log-transformed continuous variable. Additionally, we categorized TSH levels in quartiles $(0.46-1.44,1.45-1.97,1.98-2.68$, and 2.69$4.68 \mathrm{mUI} / \mathrm{L})$.

To better understand the role of each metabolic syndrome factors (waist circumference, HDL levels, triglyceride levels, blood pressure, and fasting glucose), as well as other possible confounders such as BMI, physical activity, and smoking, we performed additional models for each of those predictors individually after adjustment for age and gender.

A level of significance of 0.05 was used. All analyses were performed using Stata version 13.0 (StataCorp, USA).

\section{Results}

We included 10,539 individuals, $73 \%$ male, with mean age of $43.4 \pm 9.4$ years and mean BMI of $26.4 \pm 4.2 \mathrm{~kg} / \mathrm{m}^{2}$. Baseline characteristics, according to normal range TSH quartiles, are shown in Table 1. Due to the large sample size, almost all variables were statistically different among the groups, though the absolute differences were small.

The overall prevalence of NAFLD was $37.3 \%$. Compared to individuals without NAFLD, those with the condition were older and with more metabolic abnormalities (Table 2). 
Table 1. Baseline characteristics of subjects stratified according to TSH quartiles

\begin{tabular}{|c|c|c|c|c|c|c|}
\hline Gender, male, \% & 71 & 74 & 73 & 72 & 73 & 0.04 \\
\hline Age, years & $43.04 \pm 9.25$ & $43.20 \pm 9.14$ & $43.36 \pm 9.25$ & $43.85 \pm 10.02$ & $43.36 \pm 9.43$ & 0.01 \\
\hline $\mathrm{BMI}, \mathrm{kg} / \mathrm{m}^{2}$ & $25.86 \pm 3.89$ & $26.26 \pm 3.93$ & $26.69 \pm 4.42$ & $26.72 \pm 4.39$ & $26.38 \pm 4.18$ & $<0.01$ \\
\hline $\mathrm{SBP}, \mathrm{mm} \mathrm{Hg}$ & $115 \pm 12$ & $116 \pm 12$ & $117 \pm 12$ & $116 \pm 12$ & $116 \pm 12$ & $<0.01$ \\
\hline $\mathrm{DBP}, \mathrm{mm} \mathrm{Hg}$ & $76 \pm 8$ & $76 \pm 8$ & $77 \pm 8$ & $77 \pm 8$ & $76 \pm 8$ & $<0.01$ \\
\hline Total cholesterol, mg/dL & $180 \pm 34$ & $188 \pm 35$ & $189 \pm 35$ & $190 \pm 35$ & $189 \pm 35$ & 0.04 \\
\hline LDL-c, mg/dL & $116 \pm 31$ & $116 \pm 32$ & $116 \pm 32$ & $116 \pm 32$ & $116 \pm 32$ & 0.92 \\
\hline $\mathrm{HDL}-\mathrm{c}, \mathrm{mg} / \mathrm{dL}$ & $49 \pm 14$ & $47 \pm 13$ & $48 \pm 14$ & $48 \pm 14$ & $48 \pm 14$ & $<0.01$ \\
\hline Triglycerides, mg/dL* & $98(72-140)$ & $107(75-150)$ & $109(78-156)$ & $112(81-161)$ & $108(77-153)$ & $<0.01$ \\
\hline Hypertension, $n(\%)$ & $340(12.72)$ & $378(14.47)$ & $403(15.38)$ & $387(14.69)$ & $1,508(14.31)$ & 0.04 \\
\hline Smoking, $n(\%)$ & $244(9.13)$ & $209(8.00)$ & $179(6.83)$ & $147(5.58)$ & $779(7.39)$ & $<0.01$ \\
\hline
\end{tabular}

* Triglycerides expressed as median (interquartile range); other variables expressed as mean \pm SD or $n$ (\%).

BMI, body mass index; DBP, diastolic blood pressure; HDL-c, high-density lipoprotein cholesterol; LDL-c, low-density lipoprotein cholesterol; SBP, systolic blood pressure; TSH, thyroid-stimulating hormone.

Table 2. Baseline characteristics of subjects stratified according to the presence or absence of NAFLD (mean \pm SD)

\begin{tabular}{llcc}
\hline & NAFLD & & \\
\cline { 2 - 3 } & absent & present & $p$ value \\
\hline Patients, \% & $6,607(62.7)$ & $3,932(37.3)$ & \\
Male, \% & $4,196(63.51)$ & $3,452(87.79)$ & $<0.01$ \\
Age, years & $41.73 \pm 9.20$ & $46.11 \pm 9.18$ & $<0.01$ \\
BMI, kg/m ${ }^{2}$ & $24.75 \pm 3.20$ & $29.12 \pm 4.19$ & $<0.01$ \\
Waist circumference, cm & $86.13 \pm 10.36$ & $100.74 \pm 17.43$ & $<0.01$ \\
SBP, mm Hg & $113 \pm 11$ & $121 \pm 12$ & $<0.01$ \\
DBP, mm Hg & $74 \pm 8$ & $80 \pm 8$ & $<0.01$ \\
Total cholesterol, mg/dL & $186 \pm 33$ & $193 \pm 37$ & $<0.01$ \\
LDL-c, mg/dL & $114 \pm 30$ & $119 \pm 33$ & $<0.01$ \\
HDL-c, mg/dL & $52 \pm 14$ & $42 \pm 10$ & $<0.01$ \\
Triglycerides, mg/dL* & $91(68-126)$ & $141(104-194)$ & $<0.01$ \\
Glucose, mg/dL & $85 \pm 8$ & $93 \pm 19$ & $<0.01$ \\
HbAlc, $\%$ & $5.6 \pm 0.7$ & $<0.01$ \\
Diabetes, $n$ (\%) & $59(0.89)$ & $200(5.09)$ & $<0.01$ \\
Hypertension, $n(\%)$ & $1,021(25.97)$ & $<0.01$ \\
Smoking, $n(\%)$ & $474(7.37)$ & $305(7.76)$ & 0.03 \\
\hline
\end{tabular}

* Triglycerides expressed as median (interquartile range); other variables expressed as mean \pm SD or $n$ (\%).

BMI, body mass index; DBP, diastolic blood pressure; HDL-c, high density lipoprotein cholesterol; LDL-c, low density lipoprotein cholesterol; SBP, systolic blood pressure; NAFLD, non-alcoholic fatty liver disease. 
Table 3. Logistic regression for NAFLD outcome using TSH as continuous and categorical variable

\begin{tabular}{|c|c|c|c|c|c|c|c|c|}
\hline & \multicolumn{2}{|c|}{ Univariate } & \multicolumn{2}{|c|}{ Model $1^{*}$} & \multicolumn{2}{|c|}{ Model $2^{\#}$} & \multicolumn{2}{|c|}{ Model $3^{* *}$} \\
\hline & OR & $p$ value & OR & $p$ value & OR & $p$ value & OR & $p$ value \\
\hline \multicolumn{9}{|c|}{ TSH quartiles } \\
\hline $0.46-1.44$ & 1.0 & Ref & 1.0 & Ref & 1.0 & Ref & 1.0 & Ref \\
\hline $1.45-1.97$ & 1.19 & $<0.01$ & 1.15 & 0.02 & 1.01 & 0.94 & 1.01 & 0.94 \\
\hline $1.98-2.68$ & 1.18 & $<0.01$ & 1.15 & 0.02 & 0.86 & 0.04 & 0.86 & 0.04 \\
\hline $2.69-4.68$ & 1.25 & $<0.01$ & 1.22 & $<0.01$ & 0.90 & 0.15 & 0.90 & 0.15 \\
\hline \multicolumn{9}{|c|}{ lnTSH (per one unit) } \\
\hline $0.46-4.68$ & 1.25 & $<0.01$ & 1.22 & $<0.01$ & 0.93 & 0.20 & 0.93 & 0.20 \\
\hline
\end{tabular}

* Model 1: adjusted for age and gender.

\# Model 2: adjusted for $1+$ metabolic syndrome criteria + smoking.

** Model 3: adjusted for $2+$ physical activity level + smoking.

lnTSH, log-transformed TSH (continuous variable); TSH, thyroid-stimulating hormone (mU/L); Ref, reference; NAFLD, non-alcoholic fatty liver disease.

Fig. 1. Prevalence of non-alcoholic fatty liver disease, according to thyroid-stimulating hormone quartiles (mUI/L).

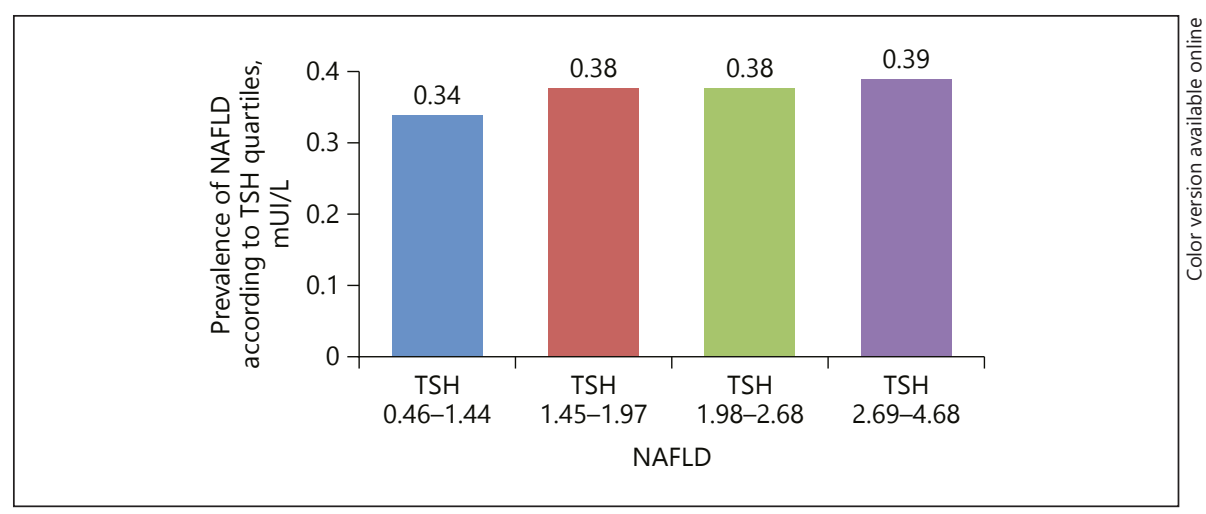

The proportion of individuals with NAFLD was higher among those with higher TSH levels (Fig. 1; $p<0.001)$. Indeed, at univariate analysis, higher TSH quartiles were associated with the diagnosis of NAFLD. This association remained significant when the model was adjusted for age and gender. However, when the data were adjusted for metabolic syndrome characteristics, the association was no longer significant, as well as when adjusted for physical activity level (Table 3 ). When TSH was modeled as a continuous logtransformed variable, the results remained unchanged (Table 3).

When further exploring the role of each individual predictor individually, the association between TSH and NAFLD lost significance after adjustment for waist circumference, BMI, and triglyceride levels, but not for other predictors (Table 4).

\section{Discussion}

This study shows an association between TSH levels within the reference range and NAFLD presence. However, this association seems to be mediated by the characteristics of metabolic syndrome (waist circumference, HDL-C levels, triglycerides levels, diabetes, and systemic arterial hypertension), as no significant association was seen after adjustment for those variables.

Many studies have investigated the role of thyroid dysfunction in the genesis of NAFLD [23, 32]. In 2012, a Korean study showed that NAFLD was statistically significantly associated with hypothyroidism and subclinical hypothyroidism in a dose-dependent manner, independently of metabolic syndrome risk factors [27]. Recently, a publication from the Rotterdam group has also found a positive association between the prevalence of NAFLD 
Table 4. Logistic regression for NAFLD outcome with individual risk factors

\begin{tabular}{|c|c|c|c|c|c|c|c|c|c|c|c|c|c|c|c|c|}
\hline & \multicolumn{2}{|c|}{$\begin{array}{l}\text { Waist } \\
\text { circumference }\end{array}$} & \multicolumn{2}{|c|}{ BMI, $\mathrm{kg} / \mathrm{m}^{2}$} & \multicolumn{2}{|c|}{ HDL } & \multicolumn{2}{|l|}{$\operatorname{lnTg}$} & \multicolumn{2}{|l|}{ SBP } & \multicolumn{2}{|c|}{ Glucose } & \multicolumn{2}{|c|}{$\begin{array}{l}\text { Physical } \\
\text { activity }^{\#}\end{array}$} & \multicolumn{2}{|c|}{ Smoking } \\
\hline & OR & $p$ value & OR & $p$ value & OR & $p$ value & OR & $p$ value & OR & $p$ value & OR & $p$ value & OR & $p$ value & OR & $p$ value \\
\hline $\operatorname{lnTSH}$ & 1.03 & 0.60 & 1.00 & 0.97 & 1.18 & $<0.01$ & 1.04 & 0.43 & 1.19 & $<0.01$ & 1.20 & $<0.01$ & 1.22 & $<0.01$ & 1.23 & $<0.01$ \\
\hline \multicolumn{17}{|c|}{ TSH quartiles } \\
\hline 1 & 1.0 & Ref & 1.0 & Ref & 1.0 & Ref & 1.0 & Ref & 1.0 & Ref & 1.0 & Ref & 1.0 & Ref & 1.0 & Ref \\
\hline 3 & 0.95 & 0.47 & 0.92 & 0.24 & 1.12 & 0.08 & 1.00 & 0.94 & 1.09 & 0.15 & 1.14 & 0.03 & 1.14 & 0.03 & 1.16 & 0.02 \\
\hline 4 & 1.00 & 0.96 & 0.98 & 0.78 & 1.17 & 0.01 & 1.02 & 0.81 & 1.19 & 0.01 & 1.20 & $<0.01$ & 1.22 & $<0.01$ & 1.23 & $<0.01$ \\
\hline
\end{tabular}

All data were adjusted for gender and age.

\# Measured by International Physical Activity Questionnaire.

BMI, body-mass index; HDL, high density lipoprotein levels; lnTg, log-transformed triglycerides levels; SBP, systolic blood pressure; ref, reference; NAFLD, non-alcoholic fatty liver disease.

and TSH levels [26]. The group was able to demonstrate that the risk of NAFLD progressively decreased from a hypothyroid to a hyperthyroid state [26]. However, in both studies, there was no analysis within the normal reference range.

In 2014, a Chinese group analyzed the prevalence of NAFLD in euthyroid subjects and found a positive association between TSH levels, even within the reference range, and NAFLD, independent of known metabolic risk factors, evaluated through multiple logistic regression [33]. However, this study evaluated less than 1,000 subjects. Our analysis has the strength of including more than 10,000 subjects and we could not demonstrate this independent association.

NAFLD is the most common liver disorder in Western industrialized countries and shares the same risk factors as type 2 diabetes, dyslipidemia, obesity, and metabolic syndrome [34]. Indeed, it may often be recognized as one of the metabolic syndrome features. Also, TSH levels have been associated with increased body fat and BMI, as well as increased cardiovascular risk [35], although the studies are still controversial [36, 37]. Within the reference range, it was already acknowledged that TSH levels seem to increase the BMI proportionally [38]. The upsurge in BMI could lead to the metabolic syndrome if other features are present and this could justify our finding of the influence of normal thyroid function and its spectrum in the prevalence of NAFLD.

The main limitation of this study is its cross-sectional design, as we cannot exclude the possibility of different results if a similar analysis was conducted consider- ing the variation of TSH levels along the time. Another point for discussion is that we only included TSH levels within the reference range, what could mitigate the expected effect of overt hypothyroidism in NAFLD. Also, as the individuals included were patients from a preventive clinic, only TSH values were available and not thyroxine levels.

Overall, our study suggests that thyroid function, even within the normal range, is associated with metabolic syndrome, through which it may play a role in NAFLD. This is an important finding because it opens a discussion if the adequacy of the TSH level to a lower reference could prevent the metabolic syndrome risk factors and, thus, the emergence of NAFLD.

\section{Disclosure Statement}

This research did not receive any specific grant from any funding agency in the public, commercial or not-for-profit sector. The authors declare no conflicts of interest.

References

1 Duntas LH: Thyroid Disease and Lipids. Thyroid. Mary Ann Liebert, Inc., 2002, vol. 12, pp 287-293.

2 Asvold BO, Bjøro T, Vatten LJ: Associations of TSH levels within the reference range with future blood pressure and lipid concentrations: 11-year follow-up of the HUNT study. Eur J Endocrinol 2013;169:73-82.

3 van Tienhoven-Wind LJ, Dullaart RP: Lownormal thyroid function and novel cardiometabolic biomarkers. Nutrients 2015;7:13521377. 
4 Crunkhorn S, Patti ME: Links between thyroid hormone action, oxidative metabolism, and diabetes risk? Thyroid. Rochelle, Mary Ann Liebert, Inc., 2008, vol. 18, pp 227-237.

5 Chubb SA, Davis WA, Davis TM: Interactions among thyroid function, insulin sensitivity, and serum lipid concentrations: the Fremantle diabetes study. J Clin Endocrinol Metab 2005; 90:5317-5320.

6 Fernández-Real JM, López-Bermejo A, Castro A, Casamitjana R, Ricart W: Thyroid function is intrinsically linked to insulin sensitivity and endothelium-dependent vasodilation in healthy euthyroid subjects. J Clin Endocrinol Metab 2006;91:3337-3343.

7 Prieur X, Huby T, Coste H, Schaap FG, Chapman MJ, Rodríguez JC: Thyroid hormone regulates the hypotriglyceridemic gene APOA5. J Biol Chem 2005;280:27533-27543.

8 Shin DJ, Osborne TF: Thyroid hormone regulation and cholesterol metabolism are connected through Sterol Regulatory ElementBinding Protein-2 (SREBP-2). J Biol Chem 2003;278:34114-34118.

9 Iglesias P, Díez JJ: Influence of thyroid dysfunction on serum concentrations of adipocytokines. Cytokine 2007;40:61-70.

10 Caldwell SH, Argo CK: Non-alcoholic fatty liver disease; in Clinical Dilemmas in NonAlcoholic Fatty Liver Disease. Chichester, John Wiley \& Sons, Ltd, 2016, pp 1-7.

11 Epidemiology of fatty liver: An update. World J Gastroenterol 2014;20:9050-9054.

12 Marchesini G, Brizi M, Bianchi G, Tomassetti S, Bugianesi E, Lenzi M, et al: Nonalcoholic fatty liver disease: a feature of the metabolic syndrome. Diabetes 2001;50:18441850.

13 Caldwell SH, Oelsner DH, Iezzoni JC, Hespenheide EE, Battle EH, Driscoll CJ: Cryptogenic Cirrhosis: Clinical Characterization and Risk Factors for Underlying Disease. Hepatology 1999;29:664-669.

14 Lefkowitch JH: Non-alcoholic fatty liver disease (NAFLD) as cause of cryptogenic cirrhosis. In: Clinical Dilemmas in Non-Alcoholic Fatty Liver Disease. Chichester, John Wiley \& Sons, Ltd, 2016, pp 36-43.

15 DI Maira G, Pastore M, Marra F: Liver fibrosis in the context of nonalcoholic steatohepatitis: the role of adipokines. Minerva Gastroenterol Dietol 2018;64:39-50.
16 Shimada M: Nonalcoholic steatohepatitis: risk factors for liver fibrosis. Hepatology Research 2002;24:429-438.

17 Villanova N, Moscatiello S, Ramilli S, Bugianesi E, Magalotti D, Vanni E, et al: Endothelial dysfunction and cardiovascular risk profile in nonalcoholic fatty liver disease. Hepatology 2005;42:473-480.

18 Luo J, Xu L, Li J, Zhao S: Nonalcoholic fatty liver disease as a potential risk factor of cardiovascular disease. Eur J Gastroenterol Hepatol 2015;27:193-199.

19 Thakur ML, Sharma S, Kumar A, Bhatt SP, Luthra K, Guleria R, et al: Nonalcoholic fatty liver disease is associated with subclinical atherosclerosis independent of obesity and metabolic syndrome in Asian Indians. Atherosclerosis 2012;223:50-511.

20 Eshraghian A: Non-alcoholic fatty liver disease and thyroid dysfunction: a systematic review. WJG 2014;20:8102-8109.

21 Lee KW, Bang KB, Rhee EJ, Kwon HJ, Lee MY, Cho YK: Impact of hypothyroidism on the development of non-alcoholic fatty liver disease: a 4-year retrospective cohort study. Clin Mol Hepatol 2015;21:372-378.

22 Parikh P, Phadke A, Sawant P: Prevalence of hypothyroidism in nonalcoholic fatty liver disease in patients attending a tertiary hospital in western India. Indian J Gastroenterol Springer India 2015;34:169-173.

23 Ludwig U, Holzner D, Denzer C, Greinert A, Haenle MM, Oeztuerk S, et al: Subclinical and clinical hypothyroidism and non-alcoholic fatty liver disease: a cross-sectional study of a random population sample aged 18 to 65 years. BMC Endocr Disord 2015;15:41.

24 Kolesnikova E, Potapenko A: Effect the cardiometabolic risk factors on vascular aging in patients with non-alcoholic fatty liver disease concomitant with subclinical hypothyroidism. Georgian Med News 2017;69-75.

25 Loria P, Carulli L, Bertolotti M, Lonardo A: Endocrine and liver interaction: the role of endocrine pathways in NASH. Nat Rev Gastroenterol Hepatol 2009;6:236-247.

26 Bano A, Chaker L, Plompen EPC, Hofman A, Dehghan A, Franco OH, et al: Thyroid function and the risk of nonalcoholic fatty liver disease: the Rotterdam Study. J Clin Endocrinol Metab 2016;101:3204-3211.

27 Chung GE, Kim D, Kim W, Yim JY, Park MJ, Kim YJ, et al: Non-alcoholic fatty liver disease across the spectrum of hypothyroidism. J Hepatol 2012;57:150-156.
28 Saunders JB, Aasland OG, Babor TF, la Fuente de JR, Grant M: Development of the Alcohol Use Disorders Identification Test (AUDIT): WHO Collaborative Project on Early Detection of Persons with Harmful Alcohol Consumption - II. Addiction 1993;88:791-804.

29 Ndumele CE, Nasir K, Conceiçao RD, Carvalho JAM, Blumenthal RS, Santos RD: Hepatic steatosis, obesity, and the metabolic syndrome are independently and additively associated with increased systemic inflammation. Arterioscler Thromb Vasc Biol 2011;31:19271932.

30 Craig CL, Marshall AL, Sjöström M, Bauman $\mathrm{AE}$, Booth ML, Ainsworth BE, et al: International physical activity questionnaire: 12-country reliability and validity. Med Sci Sports Exerc 2003;35:1381-1395.

31 Bellentani S, Saccoccio G, Masutti F, Crocè LS, Brandi G, Sasso F, et al: Prevalence of and risk factors for hepatic steatosis in Northern Italy. Ann Intern Med 2000;132:112-117.

32 Geach T: Thyroid function: poor thyroid function linked to NAFLD. Nat Rev Endocrinol 2016;12:434.

33 Tao Y, Gu H, Wu J, Sui J: Thyroid function is associated with non-alcoholic fatty liver disease in euthyroid subjects. Endocr Res 2015; 40:74-78.

34 Vernon G, Baranova A, Younossi ZM: Systematic review: the epidemiology and natural history of non-alcoholic fatty liver disease and non-alcoholic steatohepatitis in adults. Aliment Pharmacol Ther 2011;34:274-285.

35 Bano A, Dhana K, Chaker L, Kavousi M, Ikram MA, Mattace-Raso FUS, et al: Association of thyroid function with life expectancy with and without cardiovascular disease: the Rotterdam Study. JAMA Intern Med 2017; 177:1650-1657.

36 Shinkov A, Borissova A-M, Kovatcheva R, Atanassova I, Vlahov J, Dakovska L: The prevalence of the metabolic syndrome increases through the quartiles of thyroid stimulating hormone in a population-based sample of euthyroid subjects. Arq Bras Endocrinol Metabol 2014;58:926-932.

37 Laurberg P, Knudsen N, Andersen S, Carlé A, Pedersen IB, Karmisholt J: Thyroid function and obesity. Eur Thyroid J 2012;1:159-167.

38 Nyrnes A, Jorde R, Sundsfjord J: Serum TSH is positively associated with BMI. Int J Obes 2005;30:100-105. 